\title{
A 30-year-old female presenting with fever, cough and purulent sputum, and suffering from recurrent pulmonary tuberculosis
}

\section{Case history}

A 30-year-old female presented with fever, cough and purulent sputum of 6 weeks' duration.

The patient had visited the hospital 1.5 years previously because of severe cough with yellowish sputum. A chest radiograph had been performed at that time, on which the findings were found to be normal (figure 1). However, flexible bronchoscopy had revealed an accessory opening from the left main bronchus.

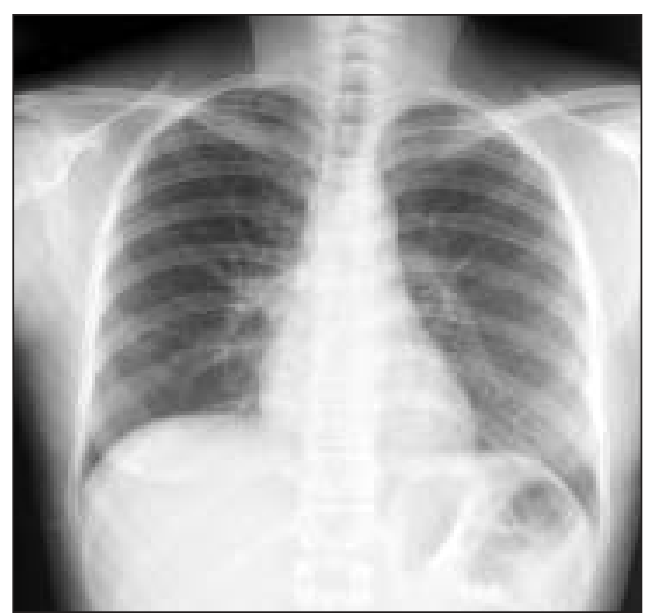

Figure 1

A chest radiograph from a 1.5-year-prior hospital admission, showing normal findings.

For the 6 months prior to the current hospital admission, the patient had been subjected to an anti-tuberculous regimen, consisting of isoniazid, rifampin, ethambutol and pyrazinamide, on an outpatient basis, as a result of pulmonary tuberculosis (TB) that was proven by a positive sputum acid-fast bacillus smear and culture. One month previously, the patient had visited another hospital, where she received antibiotic treatment for pneumonia. However, both her condition and chest radiography were aggravated in spite of the treatment. Subsequently, she was referred to Ajou University School of Medicine (Suwon, Korea) for further evaluation.

Physical examination revealed decreased breath sound on the right middle and lower lung fields. All her blood tests showed normal values, except for mild leukocytosis $\left(10,210\right.$ cells $\left.\cdot \mu \mathrm{L}^{-1}\right)$. The chest radiography obtained on admission is shown in figure 2 .

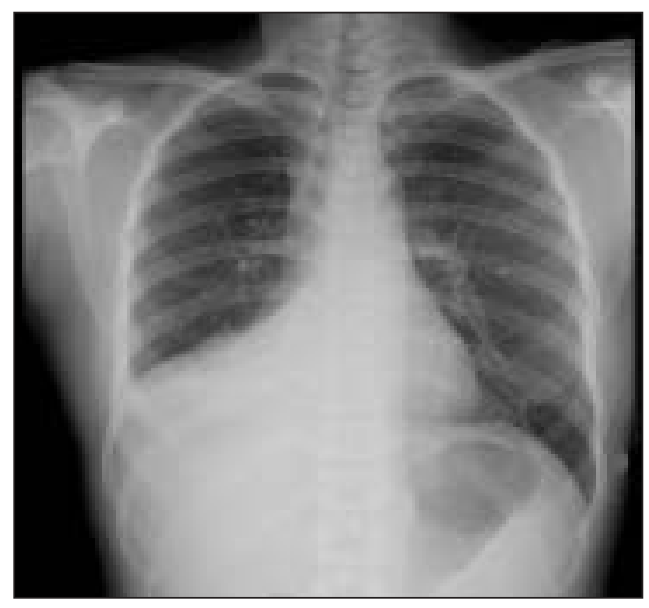

Figure 2

A chest radiograph at the time of admission.

\section{Task 1}

\section{Interpret the chest radiograph.}

Flexible bronchoscopy was also performed and the findings are shown in figure 3 below.

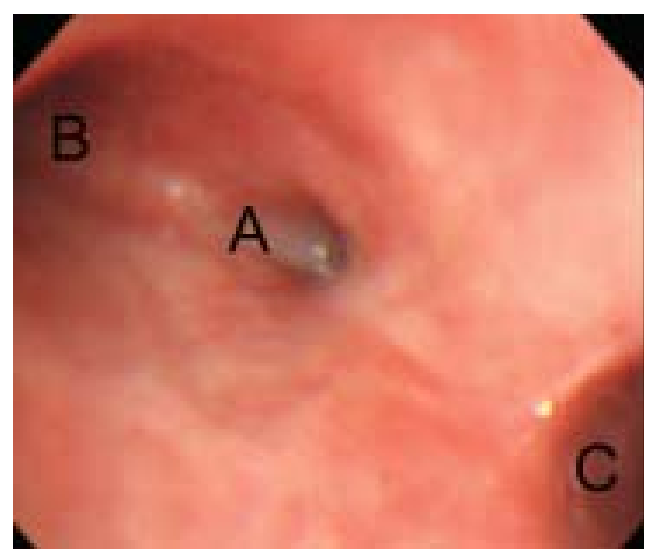

Task 2

Interpret the flexible bronchoscopy findings. What are indicated by A, B and C?
Y.J. $O h^{1}$

J.H. Park ${ }^{1}$

Y.I. Choi ${ }^{1}$

S.S. Sheen ${ }^{1}$

Y.H. Choi ${ }^{1}$

K.J. Park ${ }^{1}$

S. Lee $^{2}$

H. Choi ${ }^{2}$

K.B. Lee $^{3}$

S.C. Hwang ${ }^{1}$

Depts of ${ }^{1}$ Pulmonary and Critical Care Medicine, ${ }^{2}$ Thoracic and Cardiovascular Surgery, and ${ }^{3}$ Pathology, Ajou University School of Medicine, Suwon, Korea.

\section{Correspondence:}

S.C. Hwang

Dept of Pulmonary and Critical

Care Medicine

Ajou University School of

Medicine

San 5, Wonchon-Dong

Youngtong-Gu

Suwon 443-721

Korea

Fax: 82312195124

E-mail: schwang@ajou.ac.kr

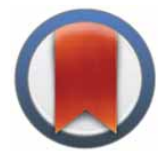

CrossMark

$\leftarrow$ click for updates

\section{Answers can be found on page 164}


Multidetector row computed tomography (MDCT), using axial, multiplanar and threedimensional volume rendering was also performed, the results of which are displayed in figure 4.
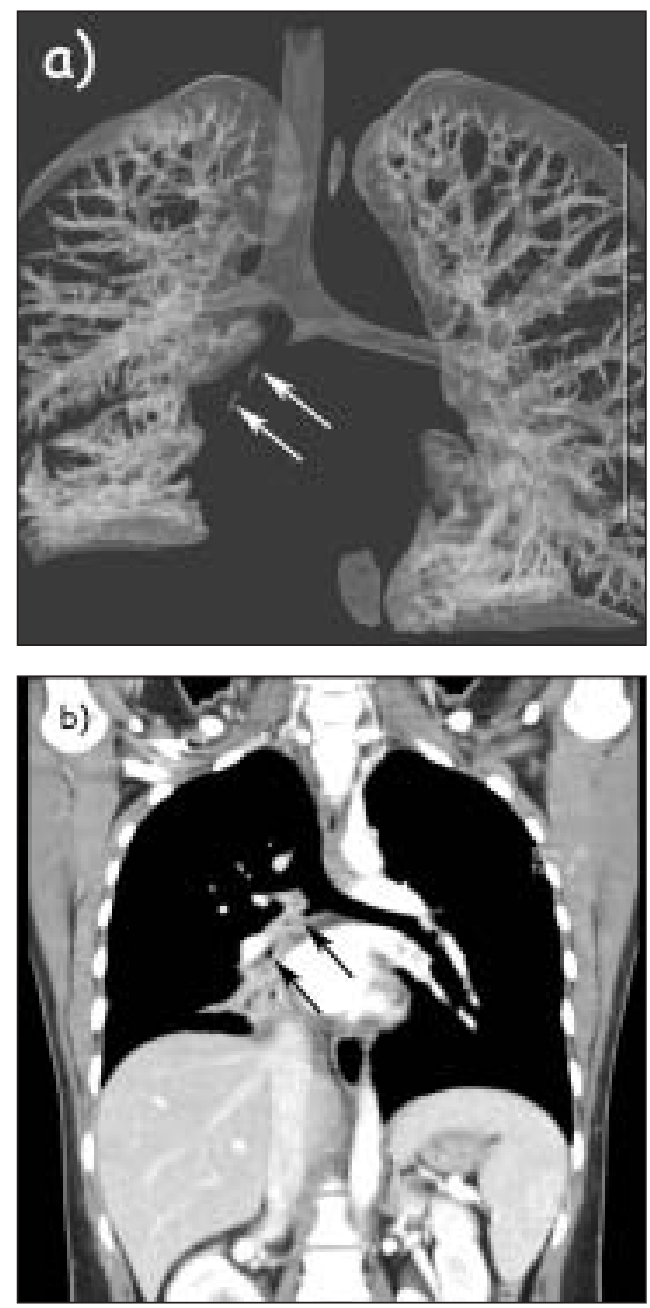

Figure 4

MDCT.

\section{Task 3}

\section{Interpret the MDCT. What do the arrows indicate?}

The patient underwent surgery due to worsening of her respiratory condition, despite antibiotic therapy, and in order to avoid recurrent infection due to poor drainage.

An end-to-side anastomosis was attempted on the right intermediate bronchus in order to salvage the collapsed right middle and lower lobes, but neither of the collapsed lobes could be reexpanded. Therefore, a bilobectomy of right middle and lower lobes was performed.
Figure 5 shows an example of both the operative and gross pathological findings from this patient.
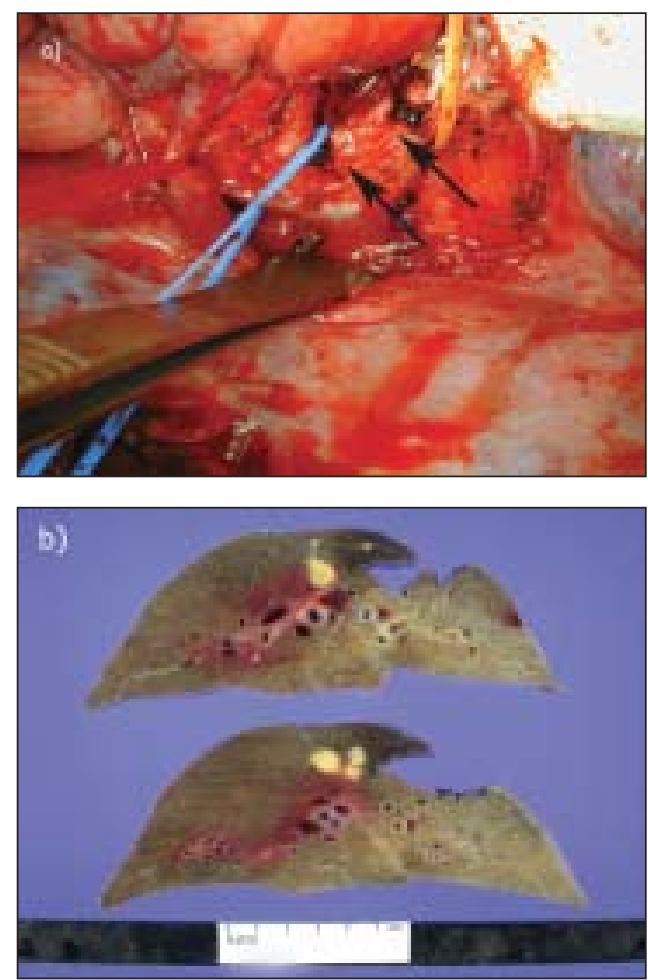

Figure 5

Operative and gross pathological findings.

\section{Task 4 \\ Interpret both the operative and gross pathological findings. What do the arrows show on the left panel?}

Microscopical pathological findings showed chronic granulomatous inflammation with caseous necrosis consistent with tuberculosis, associated bronchiectasis and submucosal dense fibrosis of the bronchi.

\section{Task 5}

Suggest a diagnosis. 


\section{Discussion}

The first reported case of bridging bronchus was diagnosed at autopsy in 1976 by Gonzalez-Crussi et al. [1]. Since then, only eight anatomically variant cases have been reported.

Bridging bronchus is usually detected during the neonatal period and is indicated by recurring atelectasis, pneumonia, bronchiectasis, chronic ventilation-perfusion mismatch, pulmonary oedema and respiratory failure [2-9]. These problems are usually caused by chronically inadequate pulmonary drainage and ventilation problems. Bridging bronchus is also often associated with a sling pulmonary artery and, occasionally, with congenital heart disease $[2,5$, $6,8,10-12]$.

Bridging bronchus is a congenital anomaly, first described as a large bronchial branch originating from the left main stem bronchus and spanning across the mediastinum from left to right, before entering the contralateral lung, as reported here $[1-4,6-8]$.

Less frequently, it is reported as a bronchial branch that crosses the mediastinum from right to left, in the opposite direction $[6,9]$. There has been one report describing a braided bronchus, in which the trachea and left bronchial tree were structurally normal and the right mainstem bronchus branched directly into four distinct segments [5].

While cases of bridging bronchus have been clinically detected in newborns, it is believed that this is the first report of an isolated bridging bronchus detected in an older patient, particularly a 30-year-old female. The longest reported surviving patient with bridging bronchus in the literature lived with neither serious symptoms nor any operative interventions for a period of only 6 months before diagnosis. This female continued to have no respiratory problems for a total of 1 year [13].

In most cases, a diagnosis of bridging bronchus is made at autopsy $[1,4,6]$, although a few ante mortem diagnoses and a successful reconstruction of the anomaly have been reported [3]. This again differs from the case described here.
The current patient had no previous medical history associated with respiratory problems before visiting the hospital. This suggests that her right intermediate bronchus did not have any drainage problems until then. No further evaluation was performed when the patient was first diagnosed with pulmonary $\mathrm{TB}$, as she was treated in another local hospital and her symptoms had gradually improved on anti-tuberculous medication. Endobronchial TB is suspected of contributing to the bronchial stricture (submucosal dense fibrosis of the bronchus from the pathological findings) that was found by MDCT and confirmed by surgery on her second visit, because, initially, at her first visit, her chest radiography showed normal findings at the time when pulmonary TB was initially detected. Subsequently, deteriorating pneumonia and abnormal findings at broncho-scopy prompted further evaluation and enabled the diagnoses of a relapse of TB and bridging bronchus.

It has been reported that cases of bridging bronchus are usually associated with sling pulmonary artery, which may cause tracheobronchial compression $[6,8]$. In addition, both congenital heart disease and gastrointestinal anomalies, such as imperforate anus $[1-3,8]$, have been reported to be associated with bridging bronchus. Pulmonary complications due to bridging bronchus, when associated with congenital heart disease, can contribute significantly to morbidity and mortality [10-12]. This patient had neither left sling pulmonary artery nor serious previous respiratory problems.

Bronchography is usually required for detecting bronchial anatomical variations of bridging bronchus, in addition to computed tomography and bronchoscopy $[5,6,13]$. However, with the introduction of MDCT, which can provide threedimensional images, information about anatomical variations of the bronchial tree were obtained without the need for bronchography.

The case presented here is unique, in that the patient maintained a normal life without any respiratory problems for 30 years, until her bridging bronchus was complicated by recurrent pulmonary TB. 


\section{Answer 1}

Chest radiography on admission displays findings consistent with right middle and lower lobar atelectasis.

\section{Answer 2}

Fibreoptic bronchoscopy shows an accessory bronchial opening from proximal left main bronchus, just below the main carina (A). Left main bronchus (B) and right main bronchus (C) are also shown. The lumen of the accessory bronchus was so narrow that the bronchoscope could not be passed through.

\section{Answer 3}

MDCT shows that the right upper lobe bronchus and right intermediate bronchus branch off from separate sites (arrows). The right intermediate bronchus arises from the left proximal main bronchus and crosses the midline, before entering the right lung, and then reaches the right middle lobe and right lower lobe. In addition, tight narrowing of the bronchus intermedius, with surrounding soft tissue mass and peripheral atelectasis, can be observed.

\section{Answer 4}

a) The operative findings, after a right thoracotomy, show the bridging bronchus (arrow) under yellow tape.

b) Gross pathological findings show several well-defined yellowish tuberculous nodules, mainly involving the lower lobe and measuring up to $1.3 \times 0.5 \mathrm{~cm}$, and in which distal bronchi are dilated. The surrounding lung parenchyma is brown, soft and unremarkable.

\section{Answer 5}

Isolated bridging bronchus associated with recurrent pulmonary tuberculosis.

\section{References}

1. Gonzalez-Crussi F, Padilla L, Miller JK, Grosfeld JL. Bridging bronchus: a previously undescribed airway anomaly. Am J Dis Child 1976; 130: 1015-1018.

2. Medina-Escobedo G, Lopez-Corella E. Sling left pulmonary artery, bridging bronchus, and associated anomalies. Am J Med Genet 1992; 44: 303-306.

3. Stokes JR, Sorkness RL, Kaplan MR, et al. The bridging bronchus - successful diagnosis and repair. Arch Otolaryngol Head Neck Surg 1997; 123: 1344-1347.

4. Bertucci GM, Dickman PS, Lachman RS, Andrews J, Paulsen P. Bridging bronchus and posterior left pulmonary artery: a unique association. Pediatr Pathol 1987; 7: 637-643.

5. Wheeler DS, Poss WB, Cocalis M, Krumwiede G, Gaston $B$. Braided bronchus: a previously undescribed airway anomaly. Pediatr Pulmonol 1998; 25: 348-351.

6. Hawass ND, Badawi M, al-Muzrakchi A, et al. Horseshoe lung: differential diagnosis. Pediatr Radiol 1990; 20: 580-584.

7. Starshak RJ, Sty JR, Woods G, Kreitzer FV. Bridging bronchus: a rare airway anomaly. Radiology 1981; 140: 95-96.

8. Wells TR, Gwinn JL, Landing BH, Stanley $P$. Reconsideration of the anatomy of sling left pulmonary artery: the association of one form with bridging bronchus and imperforate anus: anatomic and diagnostic aspects. J Pediatr Surg 1988; 10: 892-898.

9. Wells TR, Stanley P, Padua EM, Landing BH, Warburton D. Serial section-reconstruction of anomalous tracheobronchial branching patterns from CT scan images: bridging bronchus associated with sling left pulmonary artery. Pediatr Radiol 1990; 20: 444-446.

10. Stanger $P$, Lucas, RV Jr, Edwards JE. Anatomic factors causing respiratory distress in acyanotic congenital cardiac disease. Pediatrics 1969; 43: 760-769.

11. Corno A, Giamberti A, Giannico S, et al. Airway obstructions associated with congenital heart disease in infancy. J Thorac Cardiovasc Surg 1990; 99: 1091-1098.

12. Davis DA, Tucker JA, Russo P. Management of airway obstruction in patients with congenital heart defects. Ann Otol Rhinol Laryngol 1993; 102: 163-166.

13. Rishavy TJ, Goretsky MJ, Langenburg SE, Klein MD. Anterior bridging bronchus. Pediatr Pulmonol 2003; 35: 70-72. 